\title{
Positional Tracking System Using Smartphone Sensor Information
}

\author{
Jung Yee, $\mathrm{Kim}^{1 *}$
}

\begin{abstract}
The technology to locate an individual has enabled various services, its utilization has increased. There were constraints such as the use of separate expensive equipment or the installation of specific devices on a facility, with most of the location technology studies focusing on the accuracy of location verification. These constraints can result in accuracy within a few tens of centimeters, but they are not technology that can be applied to a user's location in real-time in daily life. Therefore, this paper aims to track the locations of smartphones only using the basic components of smartphones. Based on smartphone sensor data, localization accuracy that can be used for verification of the users' locations is aimed at. Accelerometers, Wifi radio maps, and GPS sensor information are utilized to implement it. In forging the radio map, signal maps were built at each vertex based on the graph data structure This approach reduces traditional map-building efforts at the offline phase. Accelerometer data were made to determine the user's moving status, and the collected sensor data were fused using particle filters. Experiments have shown that the average user's location error is about 3.7 meters, which makes it reasonable for providing location-based services in everyday life.
\end{abstract}

Key Words: Localization, Sensor fusion, WiFi Radio Map, Accelerometer.

\section{INTRODUCTION}

Location-tracking technology is a technology that detects the location of a person or object [1]. Based on this, various services such as social networking, augmented reality applications, and mobile advertising have been enabled, and their utilization is also increasing as a social safety net. Most location-checking technology studies are being carried out with the aim of implementing accuracy within a few tens of centimeters, using high-priced equipment on the side or having to install specific devices on a facility. However, for applications that are closely related to the real-life of users, it is often more important to conveniently locate and realize services that are relevant to the situation than to increase the accuracy of location using separate equipment. Accuracy within two to three meters is required in order to find a specific product at a store, but locational accuracy with a margin of error of a 10-to-30-meter radius functions for programs based on guiding system such as Pokémon GO or recommendation service system unless it is not in a congested area.

In addition, although positioning technology is a technology that requires organic connection both indoors and outdoors, Global Positioning System (GPS) technology is generalized outdoors, whereas there is a vulnerability in the interior where large loss of satellite signals can lead to very low localization accuracy or to impossibility of measurement. For this reason, indoor localization needs to be converged with other technologies.

Various technologies for indoor location tracking such as Fingerprinting using Received Signal Strength (RSS) from the WiFi or the Access Point (AP) of the mobile wireless communication network, contiguous localization using Radio-Frequency Identification (RFID) or sensor networks, and Pedestrian Dead Reckoning (PDF) using various sensors have been utilized. The most actively studied method among these is RSS-based Fingerprinting which is a method in which an administrator measures the RSS of a network AP from many of the reference point of which the locations are informed ahead in the system design process, then generates radio maps which is followed by the administrator measuring and localizing the similarity between the user-gauged RSS and radio maps.

RSS is known to perform better with more Reference Point (RP)s but accordingly, requires a considerable time to create radio maps, and the issue of exhibiting different

Manuscript received December 05, 2019; Revised December 26, 2019; Accepted December 30, 2019. (ID No. JMIS-19M-12-048) Corresponding Author (*): Jung Yee, Kim, Tongmyong University, Busan, 48520, Republic of Korea, +82-51-629-1464, kjy6858@tu.ac.kr.

${ }^{1}$ Department of Port Logistics System, Tongmyong University, Busan, 48520, Republic of Korea, kjy6858@tu.ac.kr. 
features depending on the performances and types of wireless devices' as well as the environmental changes is known to causes the inconsistent localizing performance [2]. Recently, various fusion-based localization techniques such as fusion between inertial sensor-based localization and fingerprinting are introduced to compensate for the shortcomings of fingerprinting method [3].

To locate users indoors and outdoors only with smartphones utilizing the various sensor hardware inherent in the smartphones is a technology that many applications require due to the dissemination and proliferation of smartphones [4]. Therefore, this paper makes tracking the location of the smartphone using only the basic components of smartphones that most users have the main object of study. Based on sensor data that most smartphones hold, materializing accuracy that can be used for verification of the users' locations, situation recognition, or augmented reality services is aimed at. The differentiation of this study is referred to as follows. First, in order to eliminate the disadvantages of the fingerprinting method, a graph structure that sets specific a location as the vertex is applied to measure the initial signal at the vertex position and the result is utilized in localizing to minimize the stage of radio mapping, thereby reducing the cost of time and map data construction. Second, proposing a location-tracking technique that combines data of the basic components of a smartphone which are GPS sensor, WiFi-sensor, and acceleration sensor using particle filters, location accuracy for user location-based services is provided.

The fusion of filters that can be used for location tracking and sensor data for location tracking is dealt with as related studies in Chapter 2, and Chapter 3 introduces graph-based algorithms to reduce radio mapping production and sensor data convergence algorithms using particle filters. The experiment in Chapter 4 proves its validity, and the conclusions and future plans are revealed in Chapter 5.

\section{RELATED STUDIES}

\subsection{Sensor fusion filter}

Filters used to fuse data include extended Kalman filter and particle filter.

Extended Kalman filter was developed to estimate the state vector of non-linear systems and uses Taylor series expansion to linearize the system, then estimates the state disparity vector. For location tracking which uses most inertial data, the extended Kalman filter is mostly used because nonlinear equation is applied. Because the greater the nonlinearity, the greater the error is between the Taylor series expansion approximate and true value, the sensor fusion techniques using extended Kalman filter are known to decrease in performance as the nonlinearity of the system increases [5].

Particle filter is a probabilistic algorithm of Bayes filter that estimates the state of a dynamic system from inputs with a great deal of noise [4]. It is a technique that uses sampling to estimate unknown conditions from given observational data [6]. Unlike Bayesian estimation, which calculates all states of a state vector, particle filter is realistic to utilize because it approximates the probability density distribution by using random sampling and weighting of discrete finite particles.

The posterior probability of the state $x^{t}$ estimated by the particle filter is identical to Equation (1). The normal particle filter algorithm generates new particles with weight using the measured value $z^{t}$ received from utilizing the posterior probability in the process of estimation, and normalizes the weight sum of all the particles into 1 , then uses posterior probability to create new particles. Hereafter, the process of repeating the course of re-sampling the particles so that the particles closer to the measured value are selected more frequently [7]. This study uses particle filters for sensor fusion due to their practicality.

$$
\mathrm{p}\left(x_{t} \mid z_{t}\right)=\sum_{i=1}^{N} \omega_{t}^{i} \delta\left(x_{t}-x_{t}^{i}\right)
$$

\subsection{Sensor data fusion technology}

Sensor data fusion for location tracking began with the fusion of GPS and earth magnetic field sensor data in an outdoor environment [8-9]. In general situations, GPS can provide an accurate location in real time, but if GPS is blocked for a certain period of time or tries to adjust the detection cycle of the GPS to conserve energy, it is necessary to supplement the localization gap through sensor data fusion.

Since GPS is not available in indoor environments, technologies such as WiFi, ultrasound, and UWB are applied for location tracking. With WiFi routers widely installed in most of these buildings, WiFi is the most popular technology used. Recent fusion of sensor data tends to use WiFi and PDR methods together. In [10], location estimation using WiFi, PDR, and barometric sensor data was studied. In [11], gyro sensor, RSS of WLAN, and fingerprinting of indoor map information are implemented and so are particle filters in [12], using accelerometer and WLAN RSS map information.

In this paper, unlike the existing studies that utilize additional equipment and smart sensors, acceleration sensors, WiFi RSS and GPS sensor data are utilized among the basic sensors of smartphones to suggest a method for locating users. 


\section{TRACKING THE LOCATION OF SENSOR DATA FUSION}

There are many types of sensors built into smartphones such as acceleration sensors, compass sensors, proximity sensors, and GPS sensors. In this paper, fingerprinting system utilizing WiFi RSS data, verification of the users' movement status and moving estimate using acceleration sensor data, and GPS data to check the movement status and to locate whereabouts outside are used. LG V30 was utilized for data collection.

\subsection{Accelerator sensor data}

The acceleration sensor is one of the sensors used for the recognition of motion, which processes the output signal to measure the dynamic forces of acceleration, vibration and impact of an object. In order for the acceleration sensor to recognize movement in a 3-dimensional space, it has to be possible for it to detect acceleration from the direction of $\mathrm{x}$, $\mathrm{y}$, and $\mathrm{z}$ axes. This is a three-axis acceleration sensor. As shown in Figure 1, the three-axis acceleration sensor measures the pressure applied in the left and right directions on the $\mathrm{x}$-axis, on the $\mathrm{y}$-axis up and down, and on the $\mathrm{z}$-axis applied in the front and rear directions when facing the facade of a smartphone.

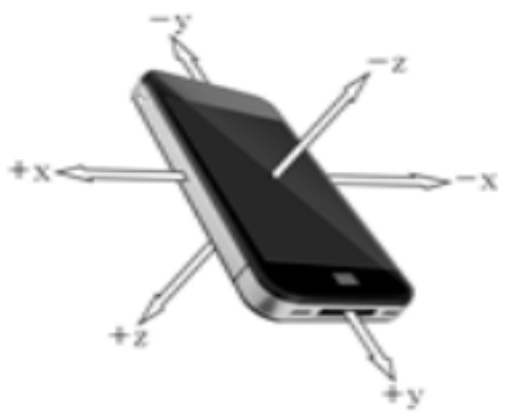

Fig. 1. 3-Axes of accelerometer.

There are many different ways to carry a smartphone, such as putting it in a pocket, carrying it in a hand, or carrying it in a bag. In addition, human motion can be classified into several forms according to its static and dynamic behavior [14]. The three-axis acceleration sensor built into a smartphone contains a rotating component, which, when the position of the smart phone changes, results in a change in the coordinate value of the acceleration data $\mathrm{x}, \mathrm{y}, \mathrm{z}$ axis, and thereby converted the acceleration sensor into a Composite Value(CV) using Equation (2) to avoid the value of these rotating components. Figure 2 shows the result of applying the measured acceleration data at every 0.001 second to Equation (2) and shows the difference between the measurement of acceleration in the stationary and moving states [14]. The figure shows that the characteristics of the measured values in the moving and stationary states are significantly different. The experiment collected and compared the data when the user stood motionless and when walking [4]. $\mathrm{x}, \mathrm{y}, \mathrm{z}$ is the acceleration data currently collected and $x^{\prime}, y^{\prime}, z^{\prime}$ is the acceleration data from the previous state:

$$
\mathrm{CV}=\sqrt{\left(x-x^{\prime}\right)^{2}+\left(y-y^{\prime}\right)^{2}+\left(z-z^{\prime}\right)^{2}} .
$$

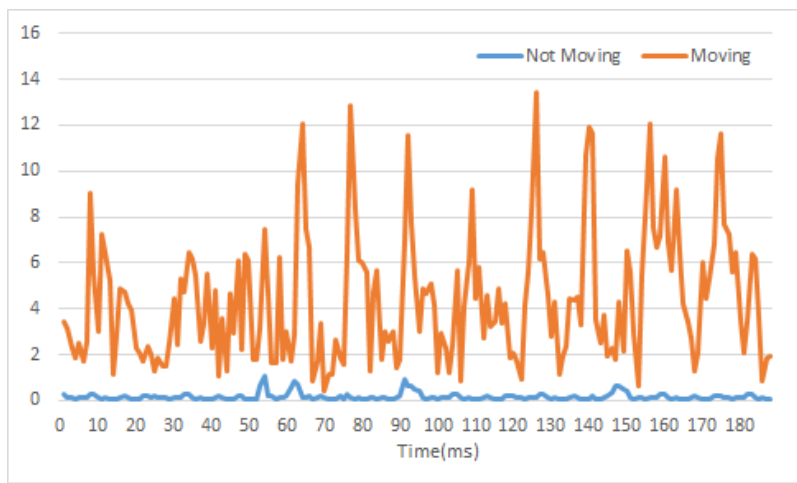

Fig. 2. Comparison of accelerometer data.

\subsection{Radio map construction}

Among WiFi-based indoor localization techniques, fingerprinting is a common technology with high precision. However, the initial radio map construction and subsequent renewal process are also technologies with disadvantages that result in a lot of labor, time and cost [13]. In this paper, several points are going to be designated to improve the mentioned shortcomings by using only the received signal strength data measured at the very point. To do this, a graph structure is used. The vertex of the graph represents the position of the signal reference, and the edge represents the structure of the traffic of the user in the building. Buildings requiring indoor positioning are complicated by obstacles that cannot be passed by people, and people will only travel to places where they can be passed, the points of intersection is set as vertices and a graphical structure will be constructed that represents the passageways between vertices with the edges. Figure 3 shows the reference position on the interior map of the building, and the area marked by the edges is calculated using the measured receiving strength at the point indicated by the vertices. The distance between the reference positions does not exceed 10 meters. Signal measurements at each point were taken 100 times in four directions, and the average signal strength was 
used to avoid using many computations for location detection. This method will reduce the initial stage of creating radio maps, which leads to saving the time cost of building a large radio map.

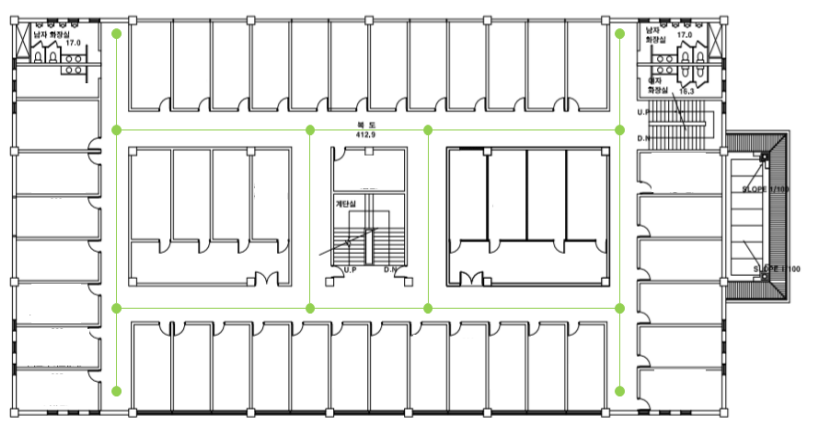

Fig. 3. Space for WiFi RSS data collection.

\subsection{Location tracking Algorithm}

Unlike most sensor fusion algorithms, which use each sensor data to estimate the position, and then converge the results, this paper has designed ones that let each measured sensor data be fused at the particle filter stage to estimate the position. Compared to the technique of fusing positional information estimated by just one sensor data, estimates by all the data available for estimation will increase the accuracy of positional location. The proposed algorithm is as shown in Figure 4.

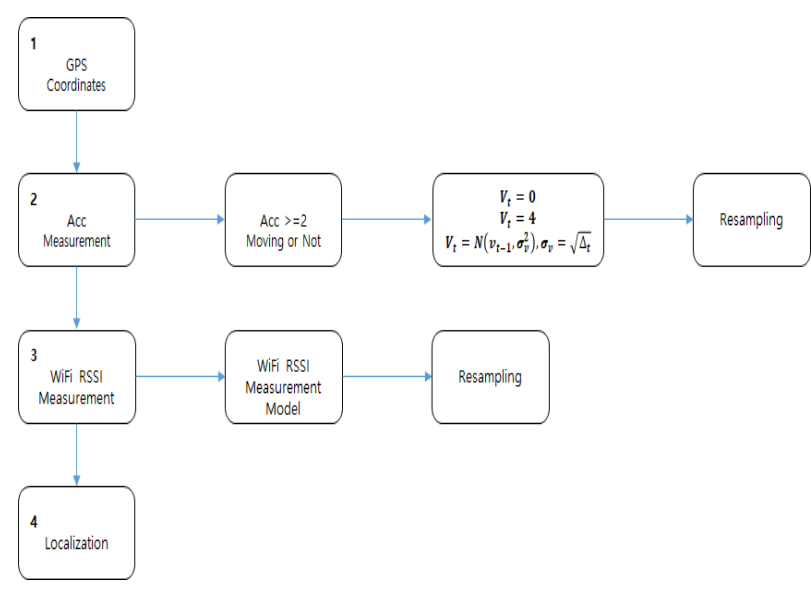

Fig. 4. Localization algorithm.

Phase 1 is the first step to measure GPS coordinates for localization, and when the coordinates are measured, they indicate the user's location. When generally using GPS coordinates, it is known that the localization error outside is as high as tens of meters, and the coordinates were not readable in the building congested areas in the process of this study either. However, this research's algorithm is able to continue localizing by using other sensors when receiving GPS coordinates is disconnected.

If location verification cannot be completed by Phase 1, discerning the user's movement using the collected acceleration sensor data is practiced in Phase 2. The accelerometer sensor data were applied to Equation (2) to utilize the critical value 2 and to distinguish and localize the status of movement or halt.

If it is not possible to receive GPS sensor data, the WiFi radio map data and the acceleration sensor data are combined to be measured under the assumption of being indoor. For speed estimates, Equation (3) that had been utilized in [7] was used. $v_{t-1}, v_{t}$ are the previous speeds and the speeds to be estimated respectively, and the speeds are estimated using the Gaussian distribution. The accelerometers used a speed of $0(\mathrm{~km} / \mathrm{h})$ as $v_{t-1}$ when the user is stationary and an average walking speed of $4(\mathrm{~km} / \mathrm{h})$ as it when moving from a stationary state:

$$
v_{t}=N\left(v_{t-1}, \sigma_{v}^{2}\right), \quad \sigma_{v}=\sqrt{\Delta_{t}}
$$

Phase 3 is to measure the strength of a WiFi signal. The measured data are used for comparison measures with the forged radio map data and for re-sampling the particles. In other words, using sensor values, the similarity is calculated via the WiFi radio map, and this similarity is used as a weight to sample the particles. Similarity was conducted by utilizing minimum mean square error as shown in Equation (4). In Equation (4), LOF is a similarity function, and $x_{t}^{i}$ is the signal reception strength of the currently measured $i-t h$ AP, the $z^{i}$ means signal reception strength of the i-th AP stored in the map data, and $\mathrm{n}$ is the number of reference positions.

$$
\mathrm{LOF}=1 / \frac{\sum\left(x_{t}^{i}-z^{i}\right)^{2}}{n}
$$

Phase 4 is a step determining the user's position using the probabilities of the particles, and the particle with the likely probability was used as the estimated position.

\section{EXPERIMENTS AND RESULTS}

For the evaluation of the positioning algorithms proposed in this paper, the user's static and walking status were experimented and the measured positions through the user's actual location and particle filters were marked on the implementation system.

Figure 5 shows blue dots with particles placed throughout the experimental area using uniform distribution for positioning. Each of the particles randomly designates initial values for position coordinates, speed and signal strength, and is later designed to display the position modified at each unit time by posterior probability based on the collected sensor information. 


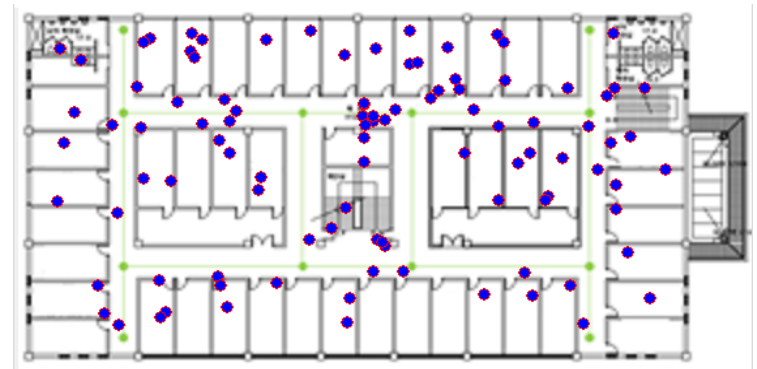

Fig. 5. Initial particle filter.

Figure 6 shows the measurement results by the algorithm of Figure 4 when the user is stationary, and is based on sensor data collected in the initial particle state on a 0.1 second basis. The actual position is indicated by a red square, a circle stands for 100 particles that the results and post probability for each particle using the value of the sensor data calculated, and one particle with the most postprobability was represented by a large circle. The red square shows the user's location.

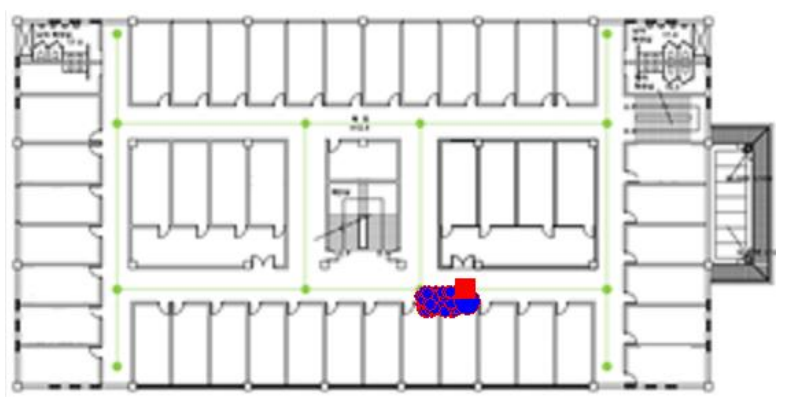

Fig. 6. Particle filter for localization when not moving.

Figure 7 is of the experimental results of when users travel. Pedestrians moved along the edges of the graph, and sensor data were also collected on a 0.1 second basis. The results show that the particles are distributed at two adjacent reference locations due to the simplification of the $\mathrm{WiFi}$ radio map, so the more accurate the spacing in radio maps, the more accurate the location will be, but the cost of deploying radio maps will also be higher.

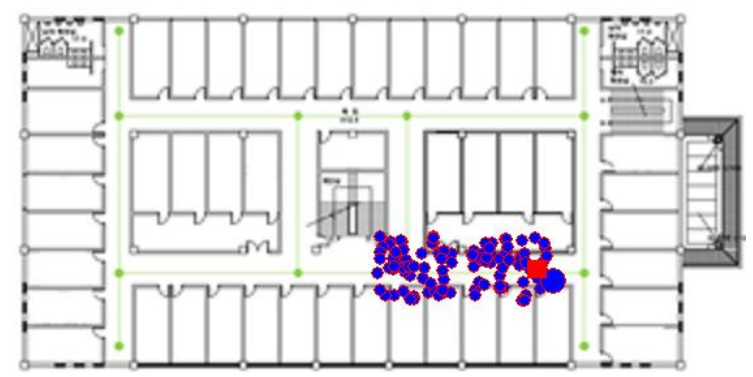

Fig. 7. Particle filter for localization when moving.

Using the same sensor data to verify the stability of the experiment and having 10 tests, the average position error of the actual position and the particle was the same as Table 1 , indicating an average position error of 3.7 meters.
Table 1. Experiment results. (unit: meter).

\begin{tabular}{c|c|c}
\hline Filter result & Not moving state & Moving state \\
\hline$\# 1$ & 1.839 & 3.645 \\
$\# 2$ & 1.699 & 3.738 \\
$\# 3$ & 1.701 & 3.596 \\
$\# 4$ & 1.914 & 3.883 \\
$\# 5$ & 1.779 & 3.741 \\
$\# 6$ & 1.688 & 3.850 \\
$\# 7$ & 1.776 & 4.060 \\
$\# 8$ & 1.883 & 3.788 \\
$\# 9$ & 1.703 & 3.668 \\
$\# 10$ & 1.587 & 3.551 \\
\hline
\end{tabular}

Figure 8 exhibits the user's tracking results. Walking along the red line was done, marking the particles with most post-probability every other second.

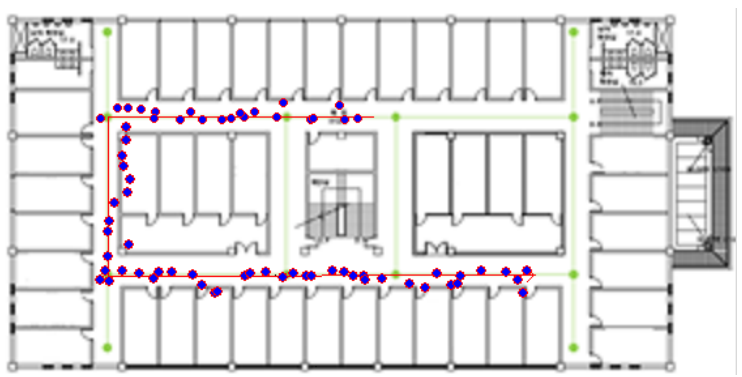

Fig. 8. Tracking result.

Table 2 is a result of a similar study that attempted to fuse sensors which shows improved accuracy when various sensors are fused for continuous location tracking indoors and outdoors. All of the results of the study presents used particle filters as filtering algorithm. [7] resulted from using smartphone sensor data only, while [12] resulted from utilizing low-cost mems accelerometers rather than smart phone sensors.

Table 2. Performance comparison with related work. (unit: meter).

\begin{tabular}{c|c|c|c}
\hline Technology & S.E & Outdoor & Indoor \\
\hline GPS & 3.08 & 2.65 & N/A \\
WiFi[7] & 3.90 & 7.24 & 5.82 \\
WiFi+accelerometer [12] & $2 \sim 6$ & & $2 \sim 6$ \\
WiFi+GSM+GPS+PDR[7] & 2.73 & 20.1 & 3.11 \\
\hline
\end{tabular}

\section{CONCLUSION}

This study provides a new location-tracking algorithm that the three types of sensor data, which are GPS sensor data for locating outdoors mainly, WiFi RSS sensor data for locating indoors, and acceleration sensor to verify user's movement status are all combined and fused with a particle filter to get locations indoors as well as outdoors in smartphone prevailing environment. Through the experiment, the smartphone's hardware specification alone solved the accuracy of the localizing was about 3.7 meters, which is available among location-related services.

The technology that verifies a user's location in real time 
using only smartphone can be used in a variety of services. Advancing from simple location-based services, the recent study on the spacetime-based application that takes into account various situations such as users' time, environment, and relationships with others is being pursued so briskly that it is expected to be utilized in various applications. This method will minimize the time and manpower required to build and manage radio maps, and will enable localization using the very smartphones that users are carrying without the need for additional expensive or special facilities, thereby enabling the rapid proliferation of the technology. Further research ought to use acceleration sensor values to not only detect the movement of users but also to measure speed, and consider fusion of real-time detection of directional transitions and earth magnetic field sensor using compass information. In addition, it is necessary to present supplementary measures for disconnect of GPS signals outdoors, and to study the possibility of more sophisticated real-time location verification by adding information on barrier walls of buildings and so on.

\section{Acknowledgement}

This research was supported by the Tongmyong University Research Grants 2017 (2017A023-1).

\section{REFERENCES}

[1] J. Hightower and G. Borriello, "Location systems for ubiquitous computing," Computers, vol. 34, no. 8, pp. 56-66, 2001.

[2] C. Laoudias, R. Pich, and C. G. Panayiotou, "Device self-calibration in location systems using signal strength histograms," Journal of Location Based Services, vol. 7, no. 3, pp. 165-181, 2013.

[3] A. Kushki, K. Plataniotis, and A. Venetsanopoulos, "Intelligent dynamic radio tracking in indoor wireless local area networks," IEEE Trans. Mobile Comput, vol. 9, no. 3, pp. 405-419, 2010.

[4] J.-Y. Kim, "Sensor fusion for seamless localization using mobile device data," Journal of the Korea Institute of Information and Communication Engineering, vol. 20, no. 10, pp. 1994-2000,

[5] S.-H. Kong, S.-Y. Jeon, and G.-H. Goh, "Current status and research trends of sensor fusion," Information \& communications magazine, vol. 32, no. 8, pp. 45-53, 2015.

[6] K.-H. Yun, D.-J. Kim, and S.-Y. Park, "Robust Location Tracking Using a Double Layered Particle Filter," Journal of Korean Institute of Information Scientists and Engineers, vol 33, no. 12, pp. 1022-1030, 2006.

[7] Maarten. Weyn, "Opportunistic Seamless Localization," Ph.D. thesis, Artesis University College of Antwerp, Belgium, 2011.

[8] Ladetto, Q. "On foot navigation: Continuous step calibration using both complementary recursive prediction and adaptive Kalman filtering," in Proceedings of the ION GPS, pp. 1735-1740, 2000.

[9] Qi. H, and Moore. J. B, "Direct Kalman filtering approach for GPS/INS integration”, IEEE Trans. Aerospace \& Electronic System, pp. 687-693, 2002.

[10] Evennou. F, Marx. F, “Advanced integration of WiFi and inertial navigation systems for indoor mobile positioning," The European Association for Signal Processing Journal on Applied Signal Processing, pp. 164-164, 2006.

[11] Leppäkoski. H, Collin. J, Takala. J, "Pedestrian navigation based on inertial sensors, indoor map, and WLAN signals," Journal of Signal Processing System, vol. 71, pp. 287-296, 2013.

[12] Wang. H, Len. H, Szabo. A, Bamberger. J, Hanebeck. U. D, "WLAN-based pedestrian tracking using particle filters and low-cost MEMS sensors," in Proceedings of the 2007 4th Workshop on Positioning, Navigation and Communication (WPNC'07), Hannover, Germany, pp. 1-7, 2007.

[13] S.-B. Yu, and W.-I. Choi, "Graph-based Wi-Fi radio map construction and update method," Journal of Korean Institute of Information Science and Engineering, vol. 44, no. 6, pp. 643-648, 2017 (in Korean).

[14] L.-K. Yang, and H.-S. Yong, "Real-time physical activity recognition using tri-axis accelerometer of smart phone," Journal of Korea Multimedia Society, vol. 17, no. 4, pp. 506-513, 2014.

\section{Author}

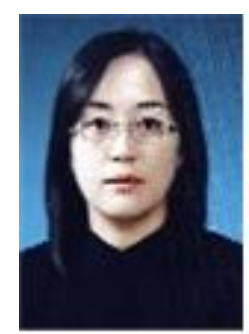

Jung-Yee Kim received her bachelor's degree in the Department of Computer Science and Statistics from the Kyungsung University in 1990. She received her MS degree in the Department of Computer Science and Statistics from Kyungsung University in 1994. She is studying for her master's degree at Pusan National University. From 2001 to 2006, she was a professor in the Department of Computer Information Processing at Tongmyong College. She has been a professor in the Department of Port Logistics System at Tongmyong University since 2006. Her current research interests include IoT and smart factory. 